\title{
Histological grades of rectal cancer: whole-volume histogram analysis of apparent diffusion coefficient based on reduced field- of-view diffusion-weighted imaging
}

\author{
Yang Peng ${ }^{1}$, Hao Tang ${ }^{1}$, Xiaoyan Meng ${ }^{1}$, Yaqi Shen ${ }^{1}$, Daoyu Hu${ }^{1}$, Ihab Kamel ${ }^{2}$, Zhen Li $^{1}$ \\ ${ }^{1}$ Tongji Hospital, Tongji Medical College of Huazhong University of Science and Technology, Wuhan 430030, China; ${ }^{2}$ Russell H. Morgan \\ Department of Radiology and Radiological Science, the Johns Hopkins Medical Institutions, Baltimore, Maryland, USA
}

Correspondence to: Zhen Li. Tongji Hospital, Tongji Medical College of Huazhong University of Science and Technology, Wuhan 430030, China. Email: zhenli@hust.edu.cn.

\begin{abstract}
Background: To explore the role of whole-lesion histogram analysis of apparent diffusion coefficient (ADC) derived from reduced field-of-view (rFOV) diffusion-weighted imaging (DWI) technique in discriminating histological grades of rectal carcinoma.
\end{abstract}

Methods: Altogether, 49 patients with rectal cancer were enrolled in this retrospective study. All patients received preoperative 3.0 T MR scan. Histogram parameters from rFOV DWI were calculated and correlated with histological differentiation of rectal cancer. The parameters were compared between different histological grades of rectal cancer by independent Student's t-test or Man-Whitney U-test. The Spearman correlation test analyzed correlations between histological grade and histogram parameters. The diagnostic performance of individual parameters for distinguishing poorly from well-/moderately differentiated tumors was assessed by receiver operating characteristic curve (ROC) analysis.

Results: There were significant differences for ADCmean, $25^{\text {th }}, 50^{\text {th }}, 75^{\text {th }}, 90^{\text {th }}, 95^{\text {th }}$ percentiles, skewness, and kurtosis of rFOV DWI sequence between well-, moderately, and poorly differentiated rectal cancers $(\mathrm{P}<0.05)$. Significant correlations were noted between histological grades and the above histogram parameters $(\mathrm{r}=0.679,0.540,0.701,0.730,0.669,0.574,-0.730$, and -0.760 respectively, $\mathrm{P}<0.001)$. Among the individual histogram parameter, kurtosis achieved the highest AUC of 0.882 with an optimal cutoff value of 1.934 in distinguishing poorly from well-/moderately differentiated rectal cancers. The combination of ADCmean, $75^{\text {th }}$ percentile, and kurtosis yielded the highest AUC of 0.927 with a sensitivity of $88.00 \%$ and a sensitivity of $91.7 \%$ using logistic regression.

Conclusions: Quantitative whole-lesion ADC histogram analysis based on the rFOV DWI technique could help differentiate histological grades of rectal cancer. The combination of ADCmean, $75^{\text {th }}$ percentile, and kurtosis may be the best choice.

Keywords: Diffusion magnetic resonance imaging (diffusion MRI); magnetic resonance imaging (MRI); gastrointestinal neoplasms; rectal neoplasms; cell differentiation

Submitted Jul 17, 2019. Accepted for publication Nov 19, 2019.

doi: 10.21037 /qims.2019.11.17

View this article at: http://dx.doi.org/10.21037/qims.2019.11.17

\section{Introduction}

Colorectal cancer is one of the most common cancers, ranking second in incidence in women and the third in men worldwide (1). The accurate evaluation of the histological differentiation of rectal cancer plays an important role in treatment planning and prediction of prognosis. The well-to-moderately differentiated rectal lesions, which are restricted within mucosa or submucosa 
without lymphovascular invasion, can be treated with endoscopic mucosal resection (EMR) or endoscopic submucosal dissection (ESD) $(2,3)$. However, the poorly differentiated rectal lesions require additional neoadjuvant chemoradiotherapy (CRT), as poor differentiation has been reported to be one risk factor for postoperative recurrence $(4,5)$. The assessment of different histological types may provide information for optimized and combined treatment, including surgery, endoscopic resection, CRT, or pelvic sidewall lymph node dissection (PSD).

At present, colonoscopy is the primary method for the screening of colorectal cancer (6-8). However, colonoscopy is an invasive examination, which is susceptible to sampling error. Therefore, resected tumor tissues obtained by endoscopic biopsy cannot reflect the entire tumor characteristics. Colonoscopy also cannot analyze locoregional lymph node pathological status $(8,9)$.

Magnetic resonance imaging (MRI) is an integral part of the multidisciplinary standardized treatment for rectal cancer (9). Preoperative MRI has been widely implemented for local staging to help determine treatment strategy and predict prognosis (10-12). Meanwhile, functional MRI, such as diffusion-weighted imaging (DWI), has been routinely utilized in standardized MRI protocol. The apparent diffusion coefficient (ADC) obtained from regions of interest (ROIs) in DWI can provide quantitative information for cancerous tissues (13). It is well known that DWI/ADC reflects different histopathological features in different tumors, and large meta-analyses (14-16) have investigated the associations between ADC, Ki 67, and cellularity in different tumors. It has been shown that different tumors demonstrate different relationships between ADCmean and/or ADCmin and expression of Ki 67. As for rectal cancer, it has been reported that the ADC values derived from the DWI technique could be useful in diagnosis, treatment, and prognosis (17-19).

Moreover, previous reports (20) have analyzed the associations between ADC histogram analysis parameters and relevant histopathological features of rectal cancer. However, many previous studies obtained ADC values from manually drawn ROIs on a single slice of the lesion, leading to subjective bias and possible sampling error of measurement. In addition, many rectal cancer lesions comprise heterogeneous components (21), so the ADC values measured on the selected ROIs cannot reflect the histological features of entire rectal tumors. The wholelesion ADC histogram analysis could capture information about the distribution of ADC measurements of the whole lesion. The utility of volume of interest (VOI) based on the entire tumor could reduce the sampling error and avoid subjective ROI selection to improve measurement reproducibility (22). For instance, the ADC histogram analysis has been used for assessing prostate cancer (23), pancreatic tumors (24), and adrenal neoplasms (25).

Furthermore, the conventional DWI technique, based on single-shot echo-planer imaging, can easily bring about distortions and artifacts (26) because of its vulnerability to off-resonance resulting from a narrow bandwidth in the phase encoding direction. However, the reduced field-ofview (rFOV) DWI, which employs 2-dimensional (2D) spatially selective echo-planar radiofrequency (RF) pulse followed by a $180^{\circ}$ refocus pulse, can provide DWI imaging with higher image quality and fewer artifacts (27-29). This technique allows for the better characterization of rectal cancer lesions by more conspicuous lesion margins and internal features (30). To date, the utility of whole-lesion histogram analysis for histological differentiation of rectal cancer based on rFOV DWI has not been well reported.

Therefore, the purpose of this study was to explore the role of whole-lesion histogram analysis based on the rFOV DWI technique in the assessment of histological differentiation of rectal cancer.

\section{Methods}

\section{Study cohort}

This retrospective study was approved by our Institutional Ethics Review Board, and informed written consent was waived. A total of 102 patients with rectal cancer were enrolled in this study from March 2016 to December 2016. The inclusion criteria were as follows: (I) patients with pathologically confirmed adenocarcinoma after surgery or endoscopy-guided biopsy; (II) patients who received $M R$, including rFOV DWI imaging. The exclusion criteria were as follows: (I) patients who received previous treatment such as CRT before MR or surgery (n=10); (II) other histological types of rectal cancer such as mucinous adenocarcinoma $(n=5)$, singlet-ring cell carcinoma $(n=3)$, or neuroendocrine tumors $(\mathrm{n}=2)$; (III) patients who could not complete the MR (n=8); (IV) patients who did not undergo surgery after MR ( $\mathrm{n}=15)$; (V) poor image quality because of obvious motion artifacts or patient movement $(n=10)$. The final study population consisted of 49 patients (34 males, 15 females; mean age, $56.2 \pm 12.2$ years; range, 22-82 years). 


\section{MRI protocols}

All MR procedures were performed using a 3T scanner (Discovery 750; GE Medical Systems Milwaukee, WI, USA) with a 32-channel torso coil. The intravenous antispasmodic agents were not administered, and patients received no bowel preparation before the MR examination. The conventional imaging protocol for all patients with rectal cancer was comprised of an axial T1 FSE sequence (TR/TE, 500/11 ms; section thickness, $3 \mathrm{~mm}$; gap, $1 \mathrm{~mm}$; FOV, $380 \times 380 \mathrm{~mm}^{2}$; flip angle, $\left.111^{\circ}\right)$; an axial T2 FSE sequence (TR/TE, 4,050/85 ms; section thickness, $3 \mathrm{~mm}$; gap, $1 \mathrm{~mm}$; FOV, $380 \times 380 \mathrm{~mm}^{2}$; flip angle, $\left.111^{\circ}\right)$; a sagittal T2 FSE sequence (TR/TE, 5,310/113 ms; section thickness, $3 \mathrm{~mm}$; gap, $0 \mathrm{~mm}$; FOV, 250×250 $\mathrm{mm}^{2}$; flip angle, $111^{\circ}$ ); and an oblique high-resolution coronal T2 FSE sequence (TR/TE, 5,629/85 ms; section thickness, $2 \mathrm{~mm}$; gap, $0 \mathrm{~mm}$; FOV, $200 \times 200 \mathrm{~mm}^{2}$; flip angle, $111^{\circ}$ ). The axial imaging was vertical to the long axis of the rectal tumor identified by the sagittal T2 sequence. The axial rFOV DWI sequence was performed for all patients. The rFOV DWI scanning parameters were as follows: TR/TE, 4,000/75 ms; section thickness, $3 \mathrm{~mm}$; gap, $0 \mathrm{~mm}$; matrix, $128 \times 64$; FOV, $200 \times 100 \mathrm{~mm}^{2}$. Two b values $\left(0\right.$ and $\left.800 \mathrm{~s} / \mathrm{mm}^{2}\right)$ were applied in 3 orthogonal directions. The scan time for the rFOV DWI sequence was 2 minutes and 32 seconds.

\section{MR image analysis}

All the image data were imported to a personal computer and analyzed by custom-developed software (Firevoxel, https:// files.nyu.edu/hr18/public/projects.html). The interpretation of all the MR images was separately performed by two radiologists with 6 years and 10 years of abdominal imaging analysis. The examiners were blinded to both clinical and pathological information of patients. The two radiologists would discuss to make final decisions by consensus. If a disagreement occurred, another radiologist with 25 years of experience aided in making the final decision.

Rectal cancer was characterized by irregular thickening of the rectal wall or intraluminal mass lesion with the intermediate high signal on T2WI image and high signal on DWI image. The examiners drew ROIs directly along the margin of the whole lesion on each slice of native DWI images $\left(b=800 \mathrm{~s} / \mathrm{mm}^{2}\right)$, by the utility of T2WI sequence as a reference standard. Specifically, the delineated ROIs covered the edge of each lesion, and ROIs were drawn along the inner margin of rectal walls to avoid the intraluminal gas, water, and other contents. Moreover, the areas of necrosis, cysts, and vessels related to each lesion at the corresponding slice were also avoided, as identified on T2WI images. In addition, the highest and lowest slices of DWI images were excluded for partial volume effects. After all the ROIs were delineated to cover the whole lesion of rectal cancer, the ADC histogram was automatically produced on a voxel-by-voxel basis along with the following corresponding parameters: ADCmean, ADCmin, $5^{\text {th }}$, $10^{\text {th }}, 25^{\text {th }}, 50^{\text {th }}$ (or median), $75^{\text {th }}, 90^{\text {th }}$, and $95^{\text {th }}$ percentiles, skewness, kurtosis, and tumor volume. The corresponding frequency table of each lesion was exported, and the above histogram parameters were computed by SPSS v. 19.0 (IBM, Armonk, NY, USA).

\section{Surgery and pathological assessment}

Forty patients underwent curative surgery (partial or total), and 9 patients had palliative operations. The median time interval between surgery and MRI scan was 3 days (range, 1-5 days). Histopathological evaluation was achieved by staining the surgical specimens with hematoxylin and eosin. The macroscopic and microscopic histological analysis of resected surgical specimens was performed by a pathologist with 25 years' experience, who was also blinded to MR imaging interpretation and related histogram analysis. The histological type and degree of differentiation of rectal cancer were assessed and recorded according to the latest WHO classification for tumors of colon and rectum (31).

\section{Statistical analysis}

The interobserver variability for histogram parameters of rFOV DWI technique was assessed using the intraclass correlation coefficient (ICC) test $(0.00-0.20$, poor agreement; $0.21-0.40$, fair agreement; $0.41-0.60$, moderate agreement; 0.61-0.80, good agreement; 0.81-1.00, excellent agreement). The Kolmogorov-Smirnov test was used to perform the normality of histogram parameters. The histogram parameters, including ADC measurements, skewness, and kurtosis, were compared between different degrees of differentiation by independent student $t$-test or the Man-Whitney U-test according to the results of the normal distribution test. The histogram parameters were adjusted for multiple testing by Bonferroni correction. The Spearman correlation analysis test was performed to assess the relationship between degrees of differentiation and histogram parameters derived from rFOV DWI $(0.00$ 
Table 1 ROC analysis of apparent diffusion coefficient histogram parameters for well/moderately vs. poorly differentiated tumors

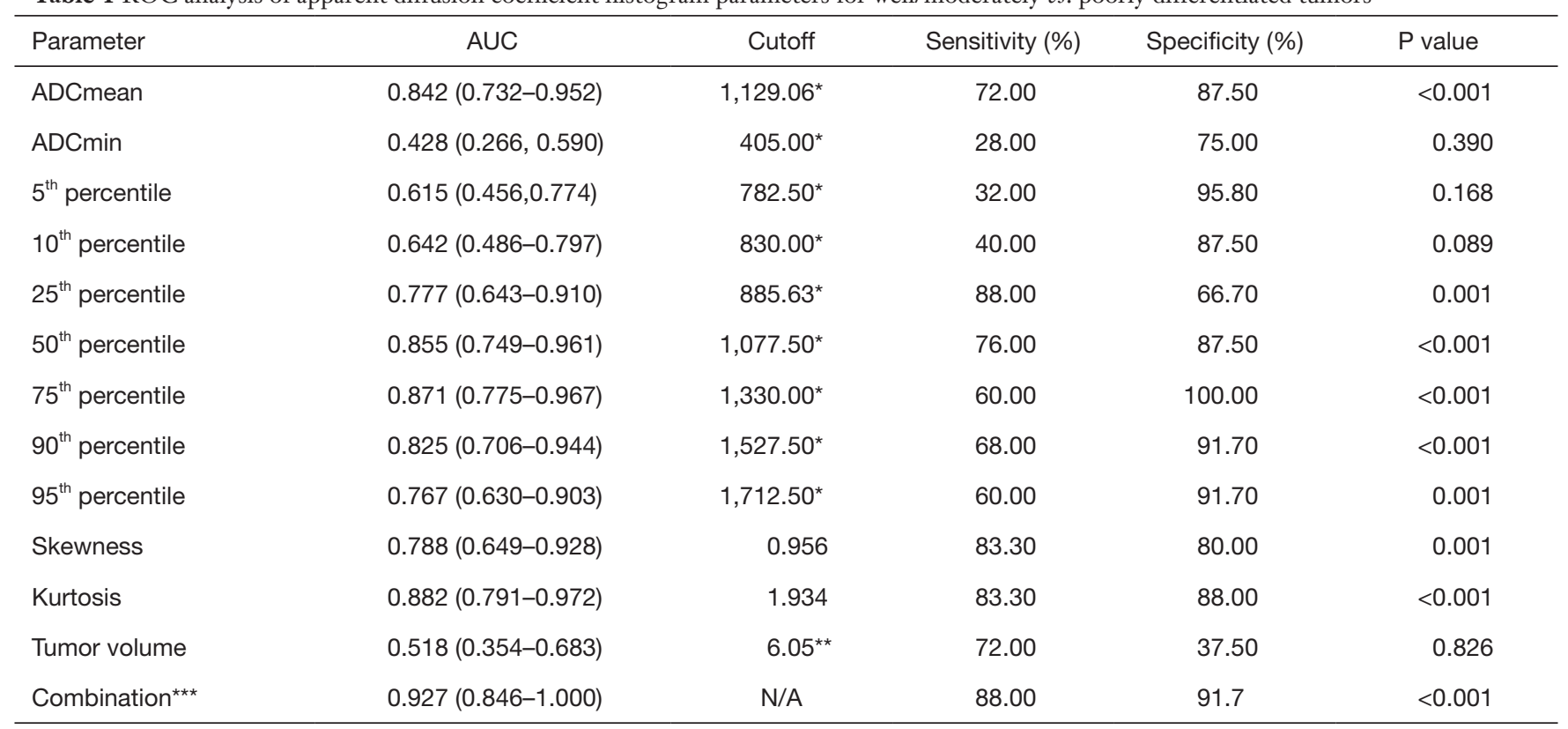

${ }^{*}$, the unit of ADC values is $10^{-6} \mathrm{~mm}^{2}$; ${ }^{* *}$, the unit of tumor volume is $\mathrm{cm}^{3}$; ${ }^{* *}$, combination of ADCmean with 75 th percentile and kurtosis.

AUC, area under the receiver operating characteristic curve; ADC, apparent diffusion coefficient.

0.19 , very weak; $0.20-0.39$, weak; $0.40-0.59$, moderate; 0.60-0.79, strong; 0.80-1.00, very strong). Receiver operating characteristic (ROC) curve analysis was applied to decide the diagnostic efficacy of histogram parameters in discriminating poorly from well-/moderately differentiated rectal adenocarcinoma. The area under the ROC curve (AUC) was examined to assess the diagnostic ability of each histogram parameter, including ADC measurements, skewness, and kurtosis. The combination of significant parameters was also studied. Because the AUCs of $75^{\text {th }}$ percentile and kurtosis were highest among individual parameters (seen in Table 1), ADCmean, $75^{\text {th }}$ percentile, and kurtosis were combined by logistic regression to obtain the highest AUC for diagnosis of histological grades of rectal cancer. The combined results of ADCmean, $75^{\text {th }}$ percentile, and kurtosis were compared to other individual parameters. All statistical analyses were performed with SPSS v. 19.0 (IBM, Armonk, NY, USA). Results with $\mathrm{P}<0.05$ were considered to have statistical significance.

\section{Results}

\section{Histopathological findings}

The patient and tumor characteristics are shown in Table 2. All 49 patients underwent surgery, and the histopathological results revealed $10(20.4 \%)$ well-, $15(30.6 \%)$ moderately, and 24 (49.0\%) poorly differentiated rectal adenocarcinomas. The tumor volume ranged from 0.94 to $31.38 \mathrm{~cm}^{3}$, and the mean tumor volume was $10.28 \pm 6.84 \mathrm{~cm}^{3}$.

\section{Interobserver agreement assessment}

The histogram parameters derived from the rFOV DWI technique between two radiologists had an excellent interobserver agreement $(\mathrm{P}<0.001$ for each parameter). The ICC values ranged from 0.970 to 0.993 (Table 3).

\section{Histogram parameters between different degrees of differentiation for rectal cancer based on the rFOV DWI technique}

The histogram parameters between different degrees of differentiation for rectal tumors based on the rFOV DWI technique are presented in Table 4. There were significant differences found in ADCmean, $25^{\text {th }}, 50^{\text {th }}$, $75^{\text {th }}, 90^{\text {th }}, 95^{\text {th }}$ percentiles, skewness, and kurtosis between well-, moderately, and poorly differentiated rectal cancers $(\mathrm{P}<0.05)$, except for $95^{\text {th }}$ percentile in the differentiation of poorly and moderately differentiated tumors ( $\mathrm{P}=0.078)$ (Figure 1). No other parameters including 
Table 2 Patient and tumor characteristics

\begin{tabular}{|c|c|}
\hline Variable & No. patient (\%) \\
\hline Age (years) & $56.2 \pm 12.2[22-82]^{*}$ \\
\hline \multicolumn{2}{|l|}{ Gender } \\
\hline Male & $34(69.4)$ \\
\hline Female & $15(30.6)$ \\
\hline \multicolumn{2}{|c|}{ Distance of primary mass from anal verge } \\
\hline $0-5.0 \mathrm{~cm}$ & $23(46.9)$ \\
\hline $5.1-10.0 \mathrm{~cm}$ & $18(36.7)$ \\
\hline $10.1-15.0 \mathrm{~cm}$ & $8(16.3)$ \\
\hline \multicolumn{2}{|l|}{ TNM stage } \\
\hline \multicolumn{2}{|l|}{ T category } \\
\hline $\mathrm{T} 1$ & $2(4.1)$ \\
\hline T2 & $19(38.8)$ \\
\hline T3 & $20(40.8)$ \\
\hline T4 & $8(16.3)$ \\
\hline \multicolumn{2}{|l|}{$\mathrm{N}$ category } \\
\hline NO & $31(63.3)$ \\
\hline $\mathrm{N} 1 / 2$ & $18(36.7)$ \\
\hline \multicolumn{2}{|l|}{ M category } \\
\hline MO & $43(87.8)$ \\
\hline M1 & $6(12.2)$ \\
\hline \multicolumn{2}{|c|}{ Histological differentiation } \\
\hline Well & $10(20.4)$ \\
\hline Moderately & $15(30.6)$ \\
\hline Poorly & $24(49.0)$ \\
\hline Tumor volume & $10.28 \pm 6.84(0.94-31.38)^{a}$ \\
\hline
\end{tabular}

${ }^{*}$, data are expressed as mean $\pm \mathrm{SD}$ with range in parenthesis; ${ }^{\mathrm{a}}$, tumor volume is given in units of $\mathrm{cm}^{3}$.

ADCmin, $5^{\text {th }}, 10^{\text {th }}$ percentiles, and tumor volume showed significant differences among rectal tumors with different differentiation degrees. Representative cases of well-, moderately, and poorly differentiated rectal cancer is shown in Figures 2-4, respectively.

Correlation of histogram parameters based on rFOV DWI with histological degrees of differentiation for rectal cancer

There were significant correlations between degrees of
Table 3 Interobserver variability of ADC histogram parameters of rFOV diffusion weighted imaging

\begin{tabular}{lc}
\hline Parameter & rFOV DWI \\
\hline ADCmean & $0.989(0.980-0.994)$ \\
ADCmin & $0.972(0.951-0.984)$ \\
$5^{\text {th }}$ percentile & $0.970(0.947-0.983)$ \\
$10^{\text {th }}$ percentile & $0.986(0.976-0.992)$ \\
$25^{\text {th }}$ percentile & $0.989(0.980-0.994)$ \\
$50^{\text {th }}$ percentile & $0.989(0.980-0.994)$ \\
$75^{\text {th }}$ percentile & $0.990(0.982-0.994)$ \\
$90^{\text {th }}$ percentile & $0.986(0.976-0.992)$ \\
$95^{\text {th }}$ percentile & $0.979(0.963-0.998)$ \\
Skewness & $0.992(0.986-0.995)$ \\
Kurtosis & $0.993(0.987-0.996)$ \\
Tumor volume & $0.992(0.986-0.996)$ \\
\hline
\end{tabular}

ADC, apparent diffusion coefficient; rFOV, reduced field-of-view; DWI, diffusion-weighted imaging.

differentiation and the following parameters: ADCmean, $25^{\text {th }}, 50^{\text {th }}, 75^{\text {th }}, 90^{\text {th }}$, and $95^{\text {th }}$ percentiles, skewness, and kurtosis $(\mathrm{P}<0.001)$ (Table 5). Among the histogram parameters, kurtosis demonstrated the strongest correlation of -0.760 with degree of differentiation (95\% CI: -0.874 to -0.575$)$, while $75^{\text {th }}$ percentile showed the highest correlation of 0.730 with degree of differentiation $(95 \%$ CI: $0.547-0.840)$.

Comparison of ROC analysis of histogram parameters in distinguishing degrees of differentiation for rectal cancer based on the rFOV DWI technique

According to the ROC analysis test from rFOV DWI, kurtosis demonstrated the highest AUC of 0.882 (95\% CI: 0.791-0.972) in discriminating poorly from well-/ moderately differentiated rectal cancer, whereas $95^{\text {th }}$ percentile achieved the lowest AUC of 0.767 (95\% CI: $0.630-0.903)$. The optimal cutoff value of kurtosis was 1.934 , with a sensitivity of $83.30 \%$ and a specificity of $88.00 \%$. The AUC for the combination of ADCmean, $75^{\text {th }}$ percentile, and kurtosis (AUC $=0.927, \mathrm{P}<0.001$ ) was found to be higher than the corresponding individual parameter, although there was no statistical significance between them (the logistic regression equation for combined parameter is as follows: combination $=-15.295+0.098 \times$ ADCmean 
Table 4 Apparent diffusion coefficient histogram parameters for degrees of histological differentiation of rectal cancer

\begin{tabular}{|c|c|c|c|}
\hline Parameter & Group & Mean & $P$ value \\
\hline \multirow[t]{3}{*}{ ADCmean } & 1 & $1,051.07$ & $0.007^{\star}$ \\
\hline & 2 & $1,127.52$ & $0.008^{\triangle}$ \\
\hline & 3 & $1,263.56$ & $<0.001^{\wedge}$ \\
\hline \multirow[t]{3}{*}{ ADCmin } & 1 & 280.83 & 0.695 \\
\hline & 2 & 257.67 & 0.565 \\
\hline & 3 & 213.00 & 0.340 \\
\hline \multirow[t]{3}{*}{$5^{\text {th }}$ percentile } & 1 & 695.30 & 0.395 \\
\hline & 2 & 714.67 & 0.490 \\
\hline & 3 & 747.05 & 0.338 \\
\hline \multirow[t]{3}{*}{$10^{\text {th }}$ percentile } & 1 & 761.88 & 0.214 \\
\hline & 2 & 788.70 & 0.181 \\
\hline & 3 & 843.15 & 0.090 \\
\hline \multirow[t]{3}{*}{$25^{\text {th }}$ percentile } & 1 & 870.05 & 0.030 \\
\hline & 2 & 921.08 & 0.029 \\
\hline & 3 & $1,010.00$ & $<0.001$ \\
\hline \multirow[t]{3}{*}{$50^{\text {th }}$ percentile } & 1 & $1,004.06$ & 0.003 \\
\hline & 2 & $1,089.33$ & 0.004 \\
\hline & 3 & $1,231.75$ & $<0.001$ \\
\hline \multirow[t]{3}{*}{$75^{\text {th }}$ percentile } & 1 & $1,182.34$ & 0.001 \\
\hline & 2 & $1,294.33$ & 0.001 \\
\hline & 3 & $1,495.00$ & $<0.001$ \\
\hline \multirow[t]{3}{*}{$90^{\text {th }}$ percentile } & 1 & $1,396.58$ & 0.014 \\
\hline & 2 & $1,514.73$ & 0.006 \\
\hline & 3 & $1,737.80$ & $<0.001$ \\
\hline \multirow[t]{3}{*}{$95^{\text {th }}$ percentile } & 1 & $1,563.76$ & 0.078 \\
\hline & 2 & $1,668.02$ & 0.029 \\
\hline & 3 & $1,881.50$ & $<0.001$ \\
\hline \multirow[t]{3}{*}{ Skewness } & 1 & 1.26 & 0.001 \\
\hline & 2 & 0.83 & 0.002 \\
\hline & 3 & 0.40 & $<0.001$ \\
\hline \multirow[t]{3}{*}{ Kurtosis } & 1 & 3.39 & 0.009 \\
\hline & 2 & 1.76 & 0.002 \\
\hline & 3 & 0.21 & $<0.001$ \\
\hline \multirow[t]{3}{*}{ Tumor volume } & 1 & 9.71 & 0.934 \\
\hline & 2 & 9.52 & 0.870 \\
\hline & 3 & 9.14 & 0.837 \\
\hline
\end{tabular}

Group 1: poorly differentiated adenocarcinoma; group 2: moderately differentiated adenocarcinoma; group 3: well differentiated adenocarcinoma. $\star$, the comparison of histogram parameters between group 1 and $2 ; \triangle$, the comparison of histogram parameters between group 2 and $3 ; \boldsymbol{\Delta}$, the comparison of histogram parameters between group 3 and 1. The mean ADC values are given the units of $10^{-} 6 \mathrm{~mm}^{2} / \mathrm{s}$. ADC, apparent diffusion coefficient.
$+0.054 \times 75^{\text {th }}$ percentile $-1.060 \times$ kurtosis). The combination of ADCmean, $75^{\text {th }}$ percentile, and kurtosis yielded a higher sensitivity $(88.00 \%)$ and specificity $(91.7 \%)$ for histological differentiation of rectal cancer (Table 1, Figure 5).

\section{Discussion}

In our study, we assessed the interobserver variability for whole-lesion histogram parameters from the rFOV DWI technique. The results showed excellent overall agreement between the two radiologists, who performed the calculation of histogram parameters from rFOV DWI. The interobserver reproducibility of 0.91 for pretreatment patients was obtained from previous study (22) of rectal cancer based on conventional DWI technique by the utility of the whole-lesion method. The value for agreement is lower than that based on the rFOV DWI technique. This phenomenon could be attributable to the use of the rFOV DWI technique, which could bring about better image quality with fewer distortions and artifacts. The excellent interobserver variability confirmed good reproducibility and stability of whole-lesion histogram analysis in combination with rFOV DWI in the assessment of rectal cancer. It is comparatively very difficult to apply DWI quantification to alimentary tracts as compared to abdominal parenchymal organs such as the liver and kidney. Based on the high-resolution rFOV DWI technique, the excellent interobserver variability of the whole-lesion histogram parameters is essential to ensure the reliability of the quantified investigation of rectal cancer.

In our study, the results demonstrated that the histogram parameters from the rFOV DWI technique could be utilized to discriminate between different degrees of differentiation for rectal cancer, including ADCmean, $25^{\text {th }}, 50^{\text {th }}, 75^{\text {th }}$, and $95^{\text {th }}$ percentiles, skewness, and kurtosis. Individually, kurtosis derived from whole-lesion histogram analysis achieved the highest AUC (0.882) in discriminating well-/moderately from poorly-differentiated rectal cancer, with the optimal cutoff value of 1.934. Furthermore, the combination of ADCmean, $75^{\text {th }}$ percentile, and kurtosis (AUC $=0.927)$ from whole-lesion histogram analysis might potentially improve the diagnostic performance in the differentiation of histological grades of rectal cancer with higher sensitivity and specificity.

Histogram parameters such as ADCmean, $25^{\text {th }}, 50^{\text {th }}$, $75^{\text {th }}, 90^{\text {th }}$, and $95^{\text {th }}$ percentiles were statistically significant in evaluating the histological grade of rectal cancer based on the rFOV DWI technique in our study. It was 

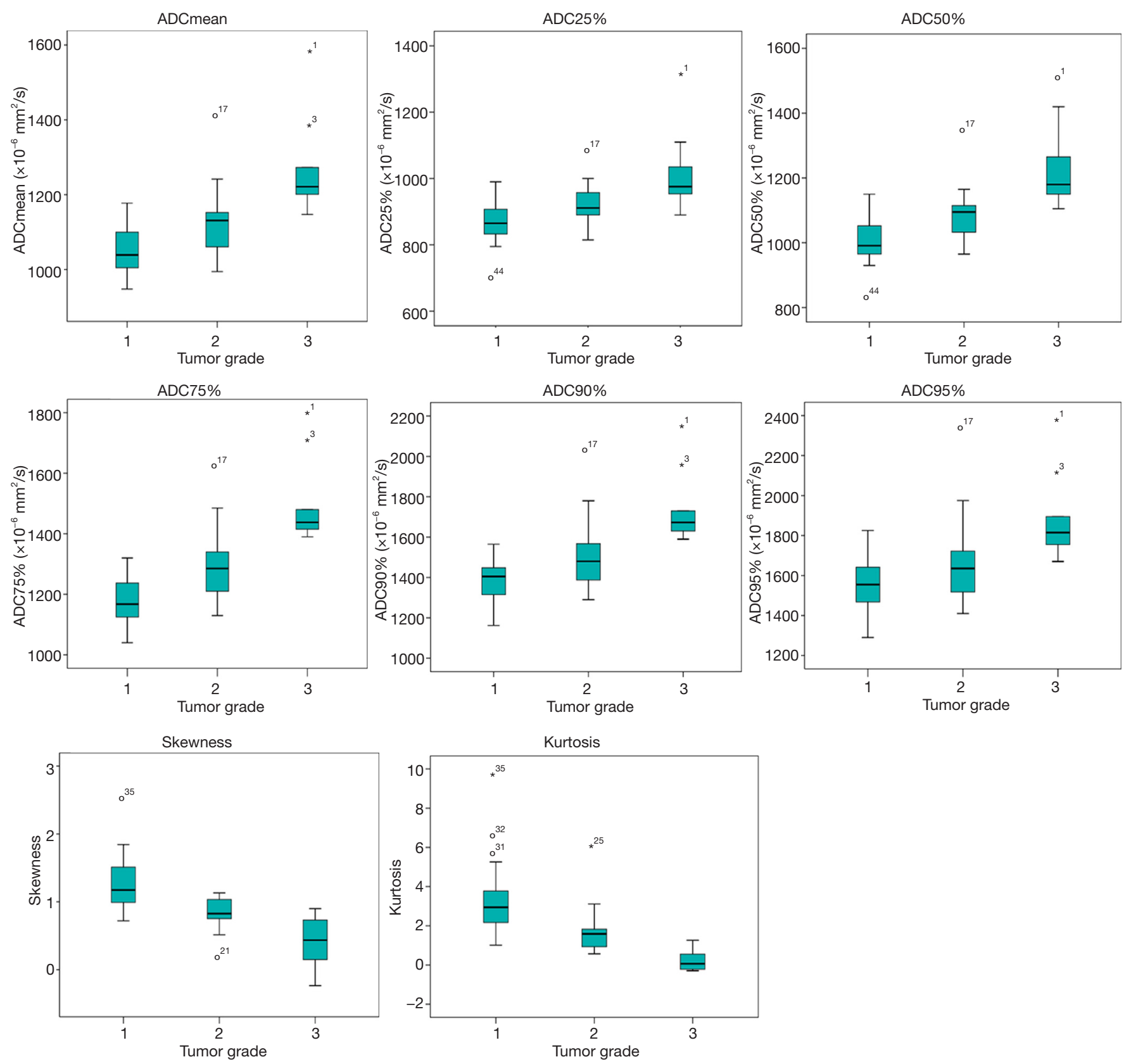

Figure 1 Boxplots exhibiting distributions of whole-lesion histogram parameters (ADC measurements, skewness, and kurtosis) based on the rFOV DWI technique in the differentiation of histological grade of rectal cancer. Tumor grade 1: poorly-differentiated; tumor grade 2: moderately differentiated; tumor grade 3: well-differentiated. ADC, apparent diffusion coefficient; rFOV, reduced field-of-view; DWI, diffusion-weighted imaging.

predominated by higher percentiles $\left(50^{\text {th }}, 75^{\text {th }}, 90^{\text {th }}\right.$, and $95^{\text {th }}$ ), which were significantly effective in the assessment of histological differentiation of rectal cancer. This finding was consistent with some previous studies. For example, both Woo et al. and Kang et al. reported that there were significant differences for high percentiles in the grading of endometrial cancer and glioma $(32,33)$. However, Zhang et al. reported that the lower percentiles $\left(5^{\text {th }}, 10^{\text {th }}\right.$, and $25^{\text {th }}$ ) exhibited better diagnostic performance in the grading of gastric cancer (34). Xue et al. found that only 

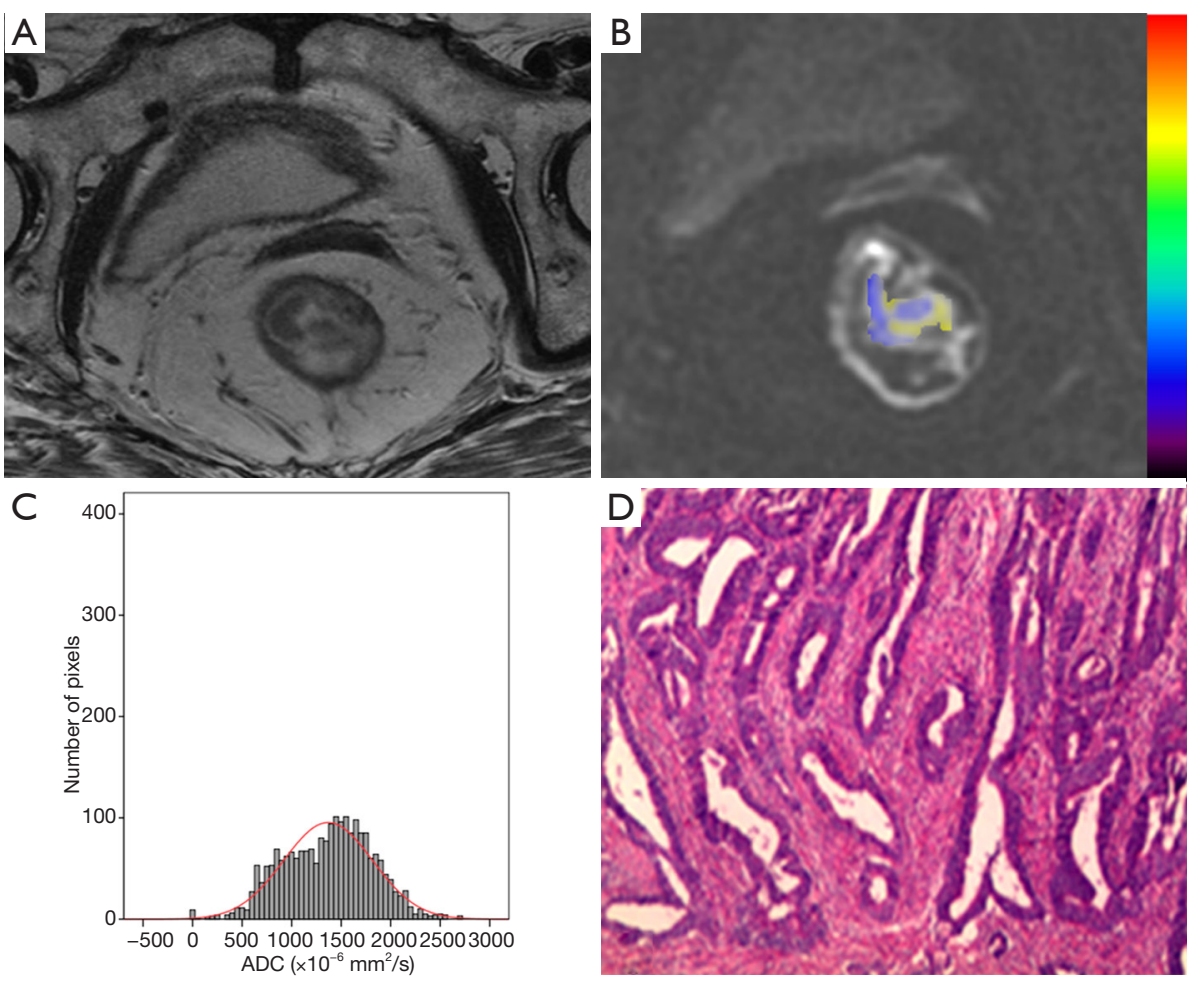

Figure 2 A 79-year-old female patient with well-differentiated rectal adenocarcinoma. (A) An axial T2-weighted image showing intraluminal lesion with intermediate signal intensity in the lower segment of the rectum. (B) The Corresponding diffusion-weighted image of rFOV DWI with the identical lesion for the reconstruction of ADC measurements. (C) A whole-lesion histogram based on the rFOV DWI technique. The ADCmean, $25^{\text {th }}, 50^{\text {th }}, 75^{\text {th }}, 90^{\text {th }}, 95^{\text {th }}$ percentiles were $1,368.25 \times 10^{-6}, 1,030.00 \times 10^{-6}, 1,410.00 \times 10^{-6}, 1,700.00 \times 10^{-6}$, $1,920.00 \times 10^{-6}$, and $2,070.00 \times 10^{-6} \mathrm{~mm}^{2} / \mathrm{s}$ respectively; skewness $=-0.138$ and kurtosis $=-0.298$. (D) Histopathological hematoxylin \& eosin staining $(\mathrm{H} \& \mathrm{E})(\times 100)$ image shows a well-differentiated adenocarcinoma invading the muscular layer of the rectal wall. ADC, apparent diffusion coefficient; rFOV, reduced field-of-view; DWI, diffusion-weighted imaging.

$\mathrm{ADC}_{5-45 \%}$ could distinguish poorly from well-/moderately differentiated cervical squamous cell carcinoma (35). The above phenomena demonstrate that ADC measurements of histogram parameters are useful tools for the quantified characterization of tumor grading. The controversy in this area is related to the significance of low or high percentile ADCs, which played various roles in the assessment of tumor biological features from diverse organs. As for rectal cancer, high percentiles were found to be significantly meaningful in the evaluation of histological grade. According to previous studies $(35,36)$, the lower percentiles indicate areas with higher cellularity and less water restriction, while the higher percentiles represent areas with necrotic and cystic components, and with fewer water molecules. One probable explanation for the findings in our study was that rectal cancers with poor differentiation degree often contain many invisible small areas of necrosis and tiny cystic changes, although the conspicuous necrotic and cystic regions were excluded for histogram analysis. The possibility that the microscopic necrotic and cystic components could be included in the selected ROIs cannot be excluded concerning the spatial resolution of DWI. The cystic and necrotic components may result in the higher frequency of high ADC voxels in contrast to well-/ moderately differentiated tumors, rendering the histological differentiation of rectal cancer possible.

Moreover, $25^{\text {th }}$ percentile derived from rFOV DWI also differed significantly different between grades of rectal cancer. One possible explanation for this result could be that as the differentiation degree decreases, the normal glandular structures and epithelium polarity are lost (37). The number and density of tumor cells increase in a disordered fashion, especially for poorly differentiated tumors, which could be characterized by the lower percentile indicating higher 

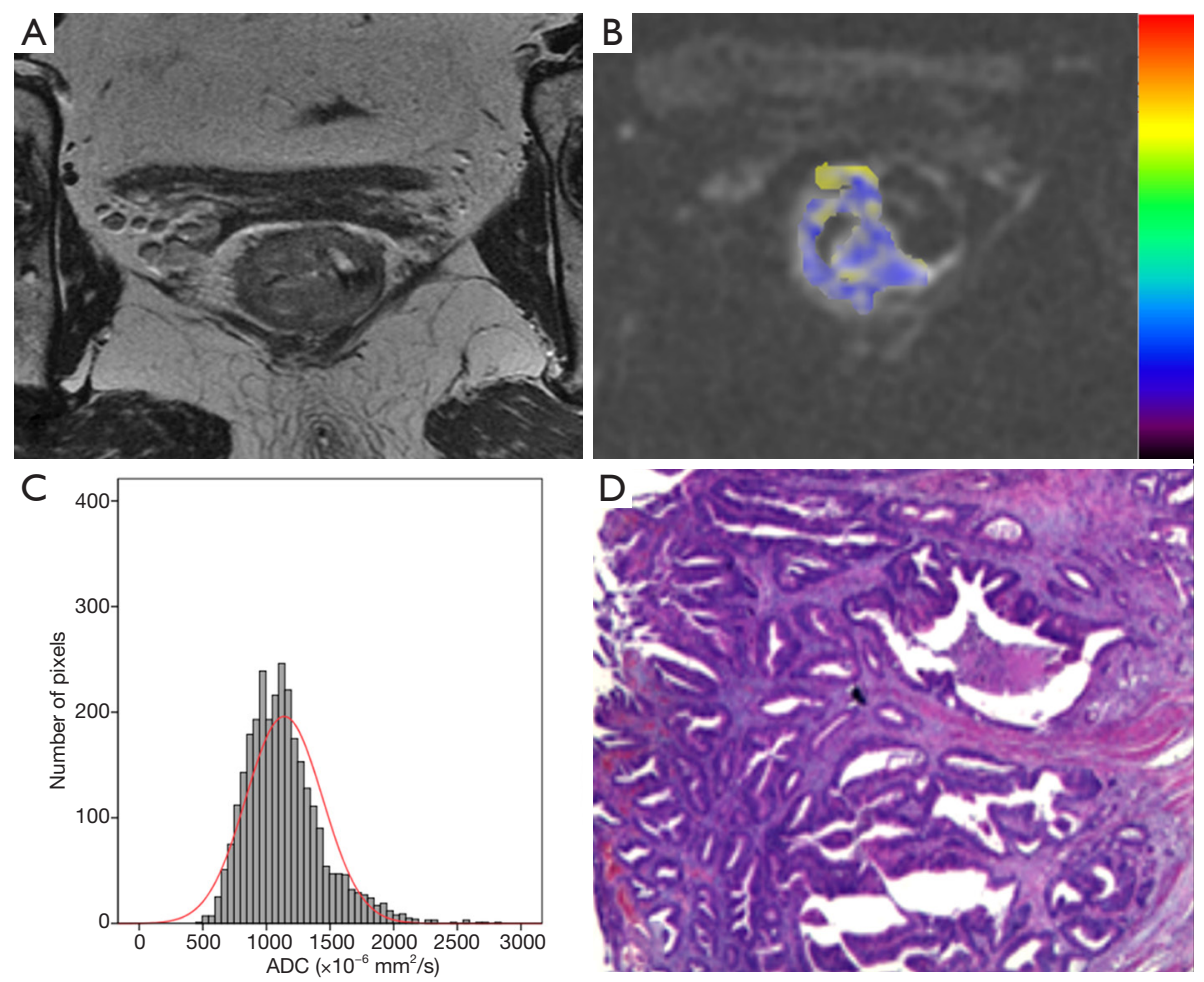

Figure 3 A 63 -year-old female patient with moderately-differentiated rectal adenocarcinoma. (A) Axial T2-weighted image showing irregular thickening of the rectal wall with intermediate signal intensity in the lower segment of the rectum. (B) The corresponding diffusion-weighted image of rFOV DWI with the same lesion for the reconstruction of ADC measurements. (C) Whole-lesion histogram based on the rFOV DWI technique. The ADCmean, $25^{\text {th }}, 50^{\text {th }}, 75^{\text {th }}, 90^{\text {th }}$ and $95^{\text {th }}$ percentiles were $1,139.74 \times 10^{-6}, 930.00 \times 10^{-6}, 1,100.00 \times 10^{-6}$, $1,290.00 \times 10^{-6}, 1,540.00 \times 10^{-6}$ and $1,730.00 \times 10^{-6} \mathrm{~mm}^{2} / \mathrm{s}$ respectively; skewness $=1.052$ and kurtosis $=1.951$. (D) Histopathological H\&E $(\times 100)$ image shows a moderate differentiated adenocarcinoma invading the whole layer of the rectal wall and surrounding adipose tissues. ADC, apparent diffusion coefficient; rFOV, reduced field-of-view; DWI, diffusion-weighted imaging.

cellularity. Moreover, the $25^{\text {th }}$ percentile might indicate the ability of the rFOV DWI technique in comparatively accurate delineation of the lesion margin, which might contain substantial non-tumor tissues such as inflammatory edema, muscle, or submucosal tissues. Adding these nontumor tissues to ADC measurements could yield high ADC values, which would affect the significance of $25^{\text {th }}$ percentile in distinguishing grades of rectal cancer. The rFOV DWI technique provided high-resolution images with fewer distortions and ghosts, rendering anatomic structures of lesions more conspicuous (30). This technique can shorten the readout time and increase the bandwidth along the band-encoding direction by reducing the number of the required k-space lines, therefore improving the image quality of the DWI image with fewer distortions and artifacts in the imaging of rectal cancer (38). These high-resolution rFOV DWI images allow more noticeable boundaries between cancer tissues and normal/inflammatory tissues, reducing the possibility of measurement biases in the delineation of margins of rectal cancers.

However, the parameter of ADCmin could not differentiate between different histological grades of rectal cancer. This may be explained by the following factors: the relatively small number of patients and the internal histopathological features of rectal cancer. Thus, it may be possible that ADCmin, and percentiles10-25 could also be sensitive to the prediction of tumor grade in rectal cancer and other kinds of tumors with an enrollment of more patients. Some previous studies $(39,40)$ indicated that ADCmin might be more sensitive than ADCmean in the reflection of cellularity/Ki 67. More future investigations are warranted to determine the role of ADCmin in the diagnosis of tumor histopathology.

Apart from the ADC percentiles we investigated, 

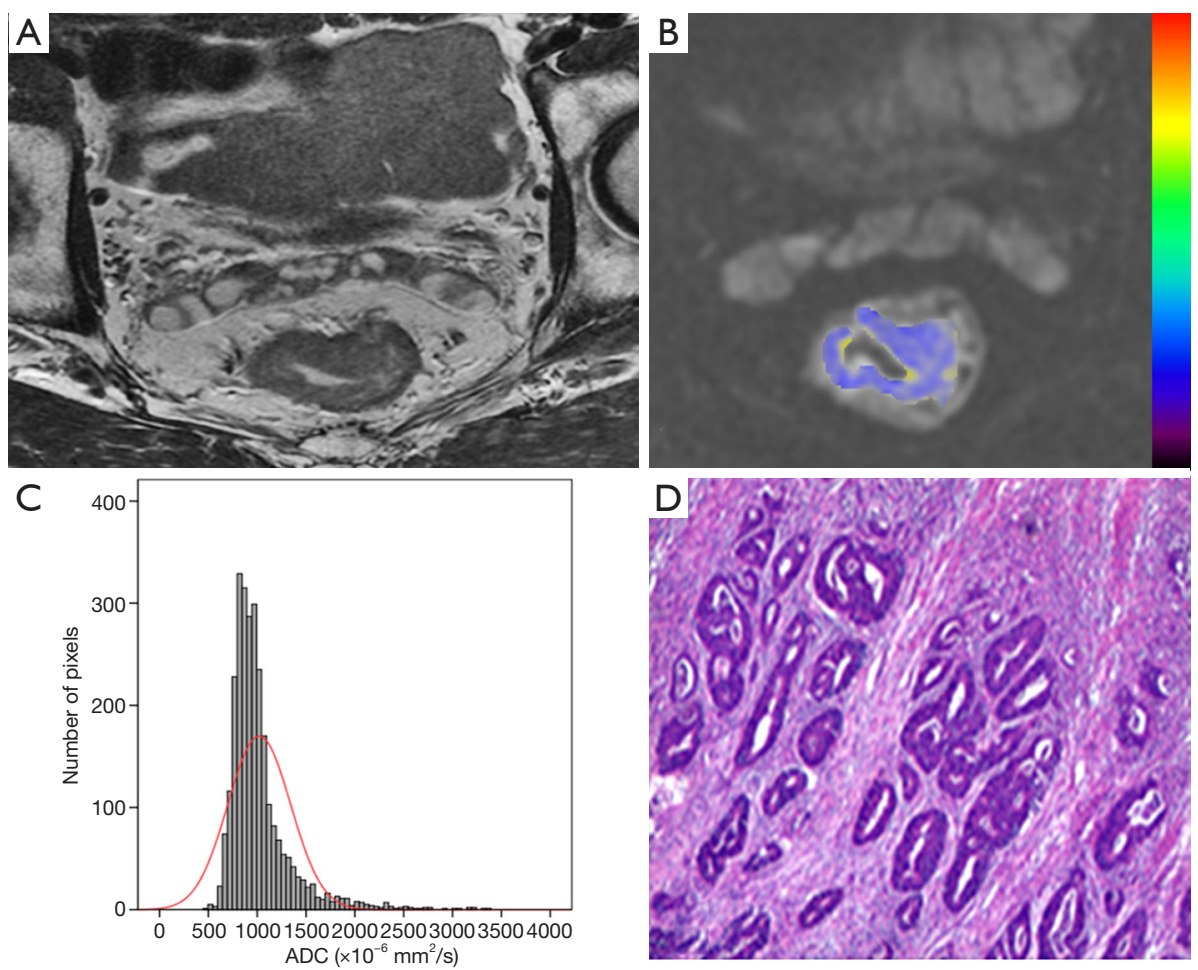

Figure 4 A 62-year-old male patient with poorly differentiated rectal adenocarcinoma. (A) An axial T2-weighted image showing irregular thickening of the rectal wall with intermediate signal intensity in the lower segment of the rectum. (B) The corresponding diffusionweighted image of rFOV DWI with the same lesion for the reconstruction of ADC measurements. (C) A whole-lesion histogram based on the rFOV DWI technique. The ADCmean, $25^{\text {th }}, 50^{\text {th }}, 75^{\text {th }}, 90^{\text {th }}$ and $95^{\text {th }}$ percentiles were $1,020.20 \times 10^{-6}, 830.00 \times 10^{-6}, 940.00 \times 10^{-6}$, $1,080.00 \times 10^{-6}, 1,370.00 \times 10^{-6}$, and $1,629.50 \times 10^{-6} \mathrm{~mm}^{2} / \mathrm{s}$ respectively; skewness $=2.682$ and kurtosis $=10.573$. (D) Histopathological H\&E $(\times 100)$ image shows a poorly differentiated adenocarcinoma invading the whole layer of the rectal wall and surrounding adipose tissues. ADC, apparent diffusion coefficient; rFOV, reduced field-of-view; DWI, diffusion-weighted imaging.

skewness and kurtosis correlated significantly with the differentiation degree of rectal cancer. In theory, kurtosis refers to the peakedness of distribution, while skewness represents the asymmetry of the distribution of grey-level intensity of pixels in a given ROI (25). Higher values of kurtosis and skewness may indicate the complexity of ROI components and increased lesion heterogeneity (21). From our study, the values of kurtosis and skewness increase as the differentiation degree of rectal cancer decreases. A possible explanation for this phenomenon could be that the poorly differentiated tumors are more often characterized by the disorganization of normal glandular structures and loss of the epithelium polarity, while as for moderately or well-differentiated rectal cancers, the amount of normal glandular structures and cell arrangements are preserved to a certain degree. The rectal cancers with a poorly differentiated degree consist of a large number of tumor cells in a disordered fashion. Their normal cellular and glandular structures are destroyed and replaced by more disproportionate tumor tissues as the differentiation degree decreases. Therefore, the values of kurtosis and skewness are negatively correlated with the histological differentiation of rectal cancer.

As for clinics, it is important to make preoperative identification of poorly from well-/moderately differentiated rectal cancers, because poorly differentiated tumors are associated with poor prognosis, including local recurrence of regional lymph nodes (4). Histogram analysis could be used to aid in the diagnosis of tumor cell differentiation and lymph node metastasis. For instance, in uterine cervical cancer, it has been shown that some ADC parameters can help distinguish tumor grades and predict lymph node metastases (41). Similar results (42) concerning lymph node metastasis were shown in thyroid cancer. However, in head 
and neck squamous cell carcinoma, $\mathrm{ADC}$ histogram analysis parameters were unable to reflect tumor grade and TNM stage (43).

In our study, the AUC values ranged from 0.767 to

Table 5 Correlation coefficients between differentiation degrees of rectal cancer and apparent diffusion coefficient parameters

\begin{tabular}{lcc}
\hline Parameter & Correlation coefficient & P value \\
\hline ADCmean & $0.679(0.469-0.809)$ & $<0.001$ \\
ADCmin & $-0.138(-0.404-0.161)$ & 0.344 \\
$5^{\text {th }}$ percentile & $0.214(-0.108-0.484)$ & 0.140 \\
$10^{\text {th }}$ percentile & $0.265(-0.020-0.519)$ & 0.065 \\
$25^{\text {th }}$ percentile & $0.540(0.321-0.720)$ & $<0.001$ \\
$50^{\text {th }}$ percentile & $0.701(0.506-0.826)$ & $<0.001$ \\
$75^{\text {th }}$ percentile & $0.730(0.547-0.840)$ & $<0.001$ \\
$90^{\text {th }}$ percentile & $0.669(0.472-0.816)$ & $<0.001$ \\
$95^{\text {th }}$ percentile & $0.574(0.326-0.741)$ & $<0.001$ \\
Skewness & $-0.730(-0.837$ to -0.558$)$ & $<0.001$ \\
Kurtosis & $-0.760(-0.874$ to -0.575$)$ & $<0.001$ \\
Tumor volume & $0.017(-0.281-0.316)$ & 0.908 \\
\hline
\end{tabular}

ADC, apparent diffusion coefficient.
0.882 , with corresponding $\mathrm{P}$ value being no more than 0.001 for the parameters ADCmean, $25^{\text {th }}, 50^{\text {th }}, 75^{\text {th }}, 90^{\text {th }}$, and $95^{\text {th }}$ percentiles, skewness, and kurtosis. The parameter of kurtosis had the largest AUC (0.882) than any other individual parameter. Hence, these parameters could be good predictive indicators for the discrimination of histological grades of rectal cancer. As for the assessment of sensitivity and specificity in our study, the sensitivity and specificity for kurtosis were $83.30 \%$ and $88.00 \%$, respectively. The sensitivities for other significant parameters ranged from $60.00 \%$ to $88.00 \%$, and the specificities ranged from $66.70 \%$ to $100.00 \%$. Most parameters were found to have comparatively higher specificities, in contrast to the lower sensitivities. In addition, the combination of ADCmean, $75^{\text {th }}$ percentile, and kurtosis yielded the highest AUC. The sensitivity and specificity were also improved, which were higher than any other individual parameter. This finding suggested that the combination of parameters might potentially improve diagnostic performance in the differentiation of histological grades of rectal cancer.

Our study had several limitations. First, this study was based on retrospective analysis with inevitable patient selection biases. Second, the sample size was relatively small. Further studies with more patients and longer time
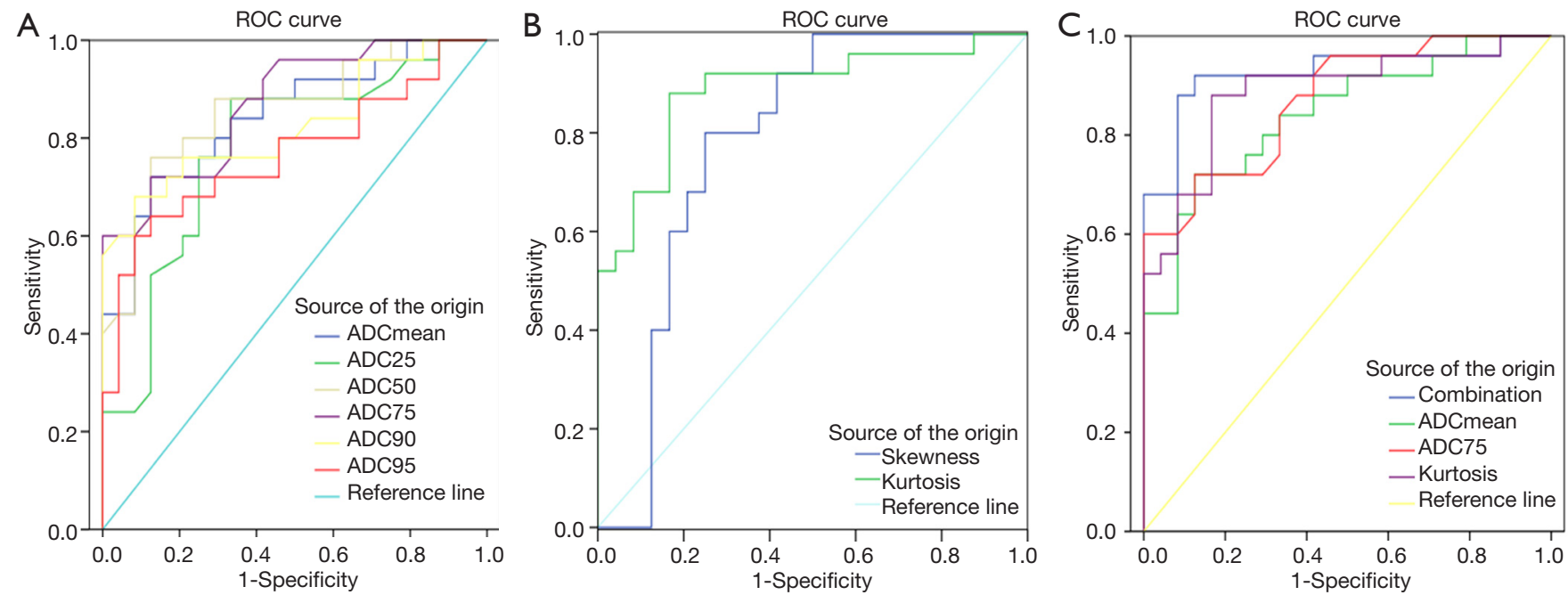

Figure 5 Receiver operating characteristic (ROC) curves demonstrating the false positive rate (sensitivity) and true positive rate (specificity) of whole-lesion histogram parameters for distinguishing well-/moderately vs. poorly differentiated rectal cancers based on the rFOV DWI technique. (A) The areas under the ROC curve (AUC) for ADCmean, $25^{\text {th }}, 50^{\text {th }}, 75^{\text {th }}, 90^{\text {th }}$, and $95^{\text {th }}$ percentiles were $0.842,0.777,0.855,0.871$, 0.825 , and 0.767 respectively. (B) The AUCs for skewness and kurtosis were 0.788 and 0.882 , respectively. (C) ROC curves for ADCmean, $75^{\text {th }}$ percentile, kurtosis, and the combination of these three parameters. Kurtosis had the largest AUC among the three individual parameters. The combination of ADCmean, $75^{\text {th }}$ percentile, and kurtosis further improved specificity and sensitivity. ADC, apparent diffusion coefficient; rFOV, reduced field-of-view; DWI, diffusion-weighted imaging. 
intervals are warranted to determine the robustness and relevance of whole-lesion histogram analysis in rectal cancer. Third, the other special histological subtypes of rectal adenocarcinoma, including mucinous adenocarcinoma and singlet-ring cell carcinoma, could also influence the ADC measurements and histogram distribution. However, we did not include these histological subtypes because most of the pathological results for rectal cancer were rectal adenocarcinoma. Finally, we only applied a b value of 0 and $800 \mathrm{~s} / \mathrm{mm}^{2}$ for analysis; however, restricted diffusion by DWI might be better characterized by higher b values. The finding of optimal maximum b value and utility of intravoxel incoherent motion are imperative to refining the results of ADC measurements.

\section{Conclusions}

In conclusion, based on the rFOV DWI technique, the whole-lesion histogram parameters could provide valuable information in the diagnosis of rectal cancer. In particular, higher percentiles, skewness, and kurtosis, might be useful indicators in the preoperative evaluation of histological differentiation of rectal cancer. The combination of ADCmean, $75^{\text {th }}$ percentile, and kurtosis may improve sensitivity and specificity. These results could be applied to clinical practice to improve the differentiation of histological features of rectal cancer by MRI.

\section{Acknowledgments}

Funding: This work was supported by the National Natural Science Foundation of China (No. 81771801, 81701657, $81501447,81571642,31630025,81320108013)$ and the Fundamental Research Funds for the Central Universities (No. 2017KFYXJJ126).

\section{Footnote}

Conflicts of Interest: The authors have no conflicts of interest to declare.

Ethical Statement: This retrospective study was approved by our Institutional Ethics Review Board, and informed written consent was waived.

\section{References}

1. Jemal A, Bray F, Center MM, Ferlay J, Ward E,
Forman D. Global cancer statistics. CA Cancer J Clin 2011;61:69-90.

2. Nakadoi K, Tanaka S, Kanao H, Terasaki M, Takata S, Oka S, Yoshida S, Arihiro K, Chayama K. Management of T1 colorectal carcinoma with special reference to criteria for curative endoscopic resection. J Gastroenterol Hepatol 2012;27:1057-62.

3. Brunner W, Widmann B, Marti L, Tarantino I, Schmied BM, Warschkow R. Predictors for regional lymph node metastasis in T1 rectal cancer: a population-based SEER analysis. Surg Endosc 2016;30:4405-15.

4. Hasegawa S, Takahashi R, Hida K, Kawada K, Sakai Y. Revisiting the treatment strategy for rectal cancer through the pattern of local recurrence. Eur J Surg Oncol 2016;42:1674-9.

5. Bianco F, Arezzo A, Agresta F, Coco C, Faletti R, Krivocapic Z, Rotondano G, Santoro GA, Vettoretto N, De Franciscis S, Belli A, Romano GM, Italian Society of Colorectal S. Practice parameters for early colon cancer management: Italian Society of Colorectal Surgery (Societa Italiana di Chirurgia Colo-Rettale; SICCR) guidelines. Tech Coloproctol 2015;19:577-85.

6. Vleugels JL, van Lanschot MC, Dekker E. Colorectal cancer screening by colonoscopy: putting it into perspective. Dig Endosc 2016;28:250-9.

7. Lieberman DA, Rex DK, Winawer SJ, Giardiello FM, Johnson DA, Levin TR. Guidelines for colonoscopy surveillance after screening and polypectomy: a consensus update by the US Multi-Society Task Force on Colorectal Cancer. Gastroenterology 2012;143:844-57.

8. Lee W, Lee D, Choi S, Chun H. Transanal endoscopic microsurgery and radical surgery for T1 and T2 rectal cancer. Surg Endosc 2003;17:1283-7.

9. Moreno CC, Sullivan PS, Mittal PK. Rectal MRI for Cancer Staging and Surveillance. Gastroenterol Clin North Am 2018;47:537-52.

10. Attenberger UI, Pilz LR, Morelli JN, Hausmann D, Doyon F, Hofheinz R, Kienle P, Post S, Michaely HJ, Schoenberg SO, Dinter DJ. Multi-parametric MRI of rectal cancer - do quantitative functional MR measurements correlate with radiologic and pathologic tumor stages? Eur J Radiol 2014;83:1036-43.

11. Taylor FG, Quirke P, Heald RJ, Moran B, Blomqvist L, Swift I, Sebag-Montefiore DJ, Tekkis P, Brown G, group Ms. Preoperative high-resolution magnetic resonance imaging can identify good prognosis stage I, II, and III rectal cancer best managed by surgery alone: a prospective, multicenter, European study. Ann Surg 2011;253:711-9. 
12. Jo SJ, Kim SH. Association between oncogenic RAS mutation and radiologic-pathologic findings in patients with primary rectal cancer. Quant Imaging Med Surg 2019;9:238-46.

13. Cao L, Chen J, Duan T, Wang M, Jiang H, Wei Y, Xia C, Zhou X, Yan X, Song B. Diffusion kurtosis imaging (DKI) of hepatocellular carcinoma: correlation with microvascular invasion and histologic grade. Quant Imaging Med Surg 2019;9:590-602.

14. Surov A, Meyer HJ, Wienke A. Associations between apparent diffusion coefficient (ADC) and KI 67 in different tumors: a meta-analysis. Part 1: ADCmean. Oncotarget 2017;8:75434-44.

15. Surov A, Meyer HJ, Wienke A. Can Imaging Parameters Provide Information Regarding Histopathology in Head and Neck Squamous Cell Carcinoma? A Meta-Analysis. Transl Oncol 2018;11:498-503.

16. Surov A, Meyer HJ, Wienke A. Correlation Between Minimum Apparent Diffusion Coefficient (ADCmin) and Tumor Cellularity: A Meta-analysis. Anticancer Res 2017;37:3807-10.

17. Curvo-Semedo L, Lambregts DM, Maas M, Beets GL, Caseiro-Alves F, Beets-Tan RG. Diffusion-weighted MRI in rectal cancer: apparent diffusion coefficient as a potential noninvasive marker of tumor aggressiveness. J Magn Reson Imaging 2012;35:1365-71.

18. Lambregts DM, Lahaye MJ, Heijnen LA, Martens MH, Maas M, Beets GL, Beets-Tan RG. MRI and diffusionweighted MRI to diagnose a local tumour regrowth during long-term follow-up of rectal cancer patients treated with organ preservation after chemoradiotherapy. Eur Radiol 2016;26:2118-25.

19. Cui Y, Yang X, Du X, Zhuo Z, Xin L, Cheng X. Wholetumour diffusion kurtosis MR imaging histogram analysis of rectal adenocarcinoma: Correlation with clinical pathologic prognostic factors. Eur Radiol 2018;28:1485-94.

20. Meyer HJ, Hohn A, Surov A. Histogram analysis of ADC in rectal cancer: associations with different histopathological findings including expression of EGFR, Hif1-alpha, VEGF, p53, PD1, and KI 67. A preliminary study. Oncotarget 2018;9:18510-7.

21. Liu L, Liu Y, Xu L, Li Z, Lv H, Dong N, Li W, Yang Z, Wang Z, Jin E. Application of texture analysis based on apparent diffusion coefficient maps in discriminating different stages of rectal cancer. J Magn Reson Imaging 2017;45:1798-808.

22. Lambregts DM, Beets GL, Maas M, Curvo-Semedo L,
Kessels AG, Thywissen T, Beets-Tan RG. Tumour ADC measurements in rectal cancer: effect of ROI methods on ADC values and interobserver variability. Eur Radiol 2011;21:2567-74.

23. Donati OF, Mazaheri Y, Afaq A, Vargas HA, Zheng J, Moskowitz CS, Hricak H, Akin O. Prostate cancer aggressiveness: assessment with whole-lesion histogram analysis of the apparent diffusion coefficient. Radiology 2014;271:143-52.

24. Li J, Liang L, Yu H, Shen Y, Hu Y, Hu D, Tang H, Li Z. Whole-tumor histogram analysis of non-Gaussian distribution DWI parameters to differentiation of pancreatic neuroendocrine tumors from pancreatic ductal adenocarcinomas. Magn Reson Imaging 2019;5 5:52-9.

25. Umanodan T, Fukukura Y, Kumagae Y, Shindo T, Nakajo M, Takumi K, Nakajo M, Hakamada H, Umanodan A, Yoshiura T. ADC histogram analysis for adrenal tumor histogram analysis of apparent diffusion coefficient in differentiating adrenal adenoma from pheochromocytoma. J Magn Reson Imaging 2017;45:1195-203.

26. Ma C, Li YJ, Pan CS, Wang H, Wang J, Chen SY, Lu JP. High resolution diffusion weighted magnetic resonance imaging of the pancreas using reduced field of view singleshot echo-planar imaging at 3 T. Magn Reson Imaging 2014;32:125-31.

27. Li A, Xing W, Li H, Hu Y, Hu D, Li Z, Kamel IR. Subtype Differentiation of Small $(</=4 \mathrm{~cm})$ Solid Renal Mass Using Volumetric Histogram Analysis of DWI at 3-T MRI. AJR Am J Roentgenol 2018;211:614-23.

28. Zaharchuk G, Saritas EU, Andre JB, Chin CT, Rosenberg J, Brosnan TJ, Shankaranarayan A, Nishimura DG, Fischbein NJ. Reduced field-of-view diffusion imaging of the human spinal cord: comparison with conventional single-shot echo-planar imaging. AJNR Am J Neuroradiol 2011;32:813-20.

29. Bhosale P, Ma J, Iyer R, Ramalingam P, Wei W, Soliman P, Frumovitz M, Kundra V. Feasibility of a reduced field-of-view diffusion-weighted (rFOV) sequence in assessment of myometrial invasion in patients with clinical FIGO stage I endometrial cancer. J Magn Reson Imaging 2016;43:316-24.

30. Peng Y, Li Z, Tang H, Wang Y, Hu X, Shen Y, Hu D. Comparison of reduced field-of-view diffusion-weighted imaging (DWI) and conventional DWI techniques in the assessment of rectal carcinoma at 3.0T: Image quality and histological T staging. J Magn Reson Imaging 2018;47:967-75.

31. Hamilton SR BF, Boffetta P et al. Tumors of the colon and 
rectum: carcinoma of the colon and rectum. In: Bosman FT, Carneiro F, Hruban RH, Theise ND (eds) World Health Organization classification of tumours. WHO classification of tumours of the digestive system, 4th edn. Lyon: IARC Press, 2010.

32. Woo S, Cho JY, Kim SY, Kim SH. Histogram analysis of apparent diffusion coefficient map of diffusionweighted MRI in endometrial cancer: a preliminary correlation study with histological grade. Acta Radiol 2014;55:1270-7.

33. Kang Y, Choi SH, Kim YJ, Kim KG, Sohn CH, Kim JH, Yun TJ, Chang KH. Gliomas: Histogram analysis of apparent diffusion coefficient maps with standard- or highb-value diffusion-weighted MR imaging--correlation with tumor grade. Radiology 2011;261:882-90.

34. Zhang Y, Chen J, Liu S, Shi H, Guan W, Ji C, Guo T, Zheng H, Guan Y, Ge Y, He J, Zhou Z, Yang X, Liu T. Assessment of histological differentiation in gastric cancers using whole-volume histogram analysis of apparent diffusion coefficient maps. J Magn Reson Imaging 2017;45:440-9.

35. Xue H, Ren C, Yang J, Sun Z, Li S, Jin Z, Shen K, Zhou W. Histogram analysis of apparent diffusion coefficient for the assessment of local aggressiveness of cervical cancer. Arch Gynecol Obstet 2014;290:341-8.

36. Shindo T, Fukukura Y, Umanodan T, Takumi K, Hakamada H, Nakajo M, Umanodan A, Ideue J, Kamimura K, Yoshiura T. Histogram Analysis of Apparent Diffusion Coefficient in Differentiating Pancreatic Adenocarcinoma and Neuroendocrine Tumor. Medicine (Baltimore) 2016;95:e2574.

37. Liu S, Guan W, Wang H, Pan L, Zhou Z, Yu H, Liu T, Yang X, He J, Zhou Z. Apparent diffusion coefficient

Cite this article as: Peng Y, Tang H, Meng X, Shen Y, Hu D, Kamel I, Li Z. Histological grades of rectal cancer: wholevolume histogram analysis of apparent diffusion coefficient based on reduced field-of-view diffusion-weighted imaging. Quant Imaging Med Surg 2020;10(1):243-256. doi: 10.21037/ qims.2019.11.17 value of gastric cancer by diffusion-weighted imaging: correlations with the histological differentiation and Lauren classification. Eur J Radiol 2014;83:2122-8.

38. Ota T, Hori M, Onishi H, Sakane M, Tsuboyama T, Tatsumi M, Nakamoto A, Kimura T, Narumi Y, Tomiyama N. Preoperative staging of endometrial cancer using reduced field-of-view diffusion-weighted imaging: a preliminary study. Eur Radiol 2017;27:5225-35.

39. Chen L, Liu M, Bao J, Xia Y, Zhang J, Zhang L, Huang $\mathrm{X}$, Wang J. The correlation between apparent diffusion coefficient and tumor cellularity in patients: a metaanalysis. PLoS One 2013;8:e79008.

40. Onishi N, Kanao S, Kataoka M, Iima M, Sakaguchi R, Kawai M, Kataoka TR, Mikami Y, Toi M, Togashi K. Apparent diffusion coefficient as a potential surrogate marker for Ki-67 index in mucinous breast carcinoma. J Magn Reson Imaging 2015;41:610-5.

41. Schob S, Meyer HJ, Pazaitis N, Schramm D, Bremicker K, Exner M, Hohn AK, Garnov N, Surov A. ADC Histogram Analysis of Cervical Cancer Aids Detecting Lymphatic Metastases-a Preliminary Study. Mol Imaging Biol 2017;19:953-62.

42. Schob S, Meyer HJ, Dieckow J, Pervinder B, Pazaitis N, Hohn AK, Garnov N, Horvath-Rizea D, Hoffmann KT, Surov A. Histogram Analysis of Diffusion Weighted Imaging at 3T is Useful for Prediction of Lymphatic Metastatic Spread, Proliferative Activity, and Cellularity in Thyroid Cancer. Int J Mol Sci 2017;18.

43. Meyer HJ, Hamerla G, Leifels L, Hohn AK, Surov A. Whole-lesion ADC histogram analysis is not able to reflect microvessel density in HNSCC. Medicine (Baltimore) 2019;98:e15520. 